\title{
Les disparités de l'épidémie à VIH au Kenya : approche géographique
}

\section{Matthieu Miralles}

\section{(2) OpenEdition}

1 Journals

\section{Édition électronique}

URL : https://journals.openedition.org/eastafrica/607

DOI : 10.4000 /eastafrica.607

ISSN : 2790-1076

\section{Éditeur}

IFRA - Institut Français de Recherche en Afrique

\section{Édition imprimée}

Date de publication : 1 juin 2008

Pagination : 1-45

ISSN : 2071-7245

\section{Référence électronique}

Matthieu Miralles, «Les disparités de l'épidémie à VIH au Kenya : approche géographique », Les

Cahiers d'Afrique de l'Est / The East African Review [En ligne], 39 | 2008, mis en ligne le 07 mai 2019, consulté le 09 décembre 2021. URL : http://journals.openedition.org/eastafrica/607 ; DOI : https:// doi.org/10.4000/eastafrica.607

Ce document a été généré automatiquement le 9 décembre 2021.

Les Cahiers d'Afrique de l'Est / The East African Review 


\title{
Les disparités de l'épidémie à VIH au Kenya : approche géographique
}

\author{
Matthieu Miralles
}

1 Singularisée par de fortes disparités spatiales et sociales, l'épidémie à VIH-Sida dans le territoire kényan est au cœur de cette lecture dont l'objectif est de mettre en lumière les différents ressorts épidémiologiques, sociaux et géographiques de la propagation de l'épidémie de Sida, ainsi que les facteurs favorisant la vulnérabilité à l'infection à VIH au sein de la population.

Quels méthodes et objectifs singularisent les recherches géographiques portant sur l'épidémie à VIH-Sida?

3 En s'appuyant sur des données biomédicales, ces recherches s'appliquent en premier lieu à apprécier dans l'espace et à toutes les échelles d'observation l'impact de l'infection, à identifier les territoires dont les populations sont infectées ou épargnées (Amat-Roze, 2003). L'objectif est ensuite d'identifier les groupes de variables qui régissent les différences d'impact et d'évolution en s'appuyant sur les discontinuités spatiales et les processus de territorialisation observés. Ces variables relèvent de trois groupes de facteurs - facteurs de transmission, d'exposition et de propagation.

Dans une deuxième étape, les approches géographiques mettent en relation les disparités de l'infection avec des facteurs de risques, puis des contextes de vie. En somme, les différentes recherches s'appuient sur le postulat que les facteurs de risques (biomédicaux et comportementaux) s'expriment différemment selon les lieux de vie et leur contexte, où se mêlent des facteurs structurels et conjoncturels qui relèvent de la géographie (mobilités, urbanisation...) des héritages historiques, de l'économique, du social, du culturel, du politique.

5 En d'autres termes, l'apport géographique conjugue synthèse des découvertes scientifiques produites par les différentes sciences et localisation, dans une perspective systémique, de l'impact de l'infection sur la population et des différents facteurs qui concurrent a sa diffusion ou a la vulnérabilité a l'infection à VIH. Cet éclairage est susceptible d'être un atout pour appréhender de façon globale les déterminants de 
l'épidémie et mettre en évidence la nécessite de réponse multisectorielle dans le cadre des politiques de prévention et de soins.

Depuis le début de l'épidémie, la diversité des contributions scientifiques a été indispensable pour comprendre la genèse, puis les modalités de la transmission et de la diffusion du VIH. La physionomie hétérogène de l'épidémie s'est ainsi profilée comme la conséquence des propriétés biologiques du germe et d'une pluralité de facteurs biomédicaux, comportementaux, sociaux et écologiques, partages par les différents pays d'Afrique subsaharienne.

7 L'infection s'y est transmise très majoritairement par voie hétérosexuelle. La transmission de la mère à l'enfant, aussi présente, est favorisée par l'absence de prévention et de prise en charge avec des traitements par AZT. Mais les écarts de séroprévalence enregistrés entre les différentes régions indiquent qu'au-delà de ces modes de transmission, une série de facteurs intriqués favorise ou limite la transmission.

8 On peut regrouper ces facteurs en deux groupes de déterminants, qui relèvent respectivement de l'exposition sexuelle au VIH ou de la transmission.

9 Pendant l'acte sexuel, la possibilité qu'une personne soit contaminée par le VIH dépend de deux probabilités : celle qu'un individu réceptif ait une relation avec un individu infecté et celle que le virus soit transmis durant cet acte sexuel-là. Le taux de propagation du VIH dans une population est le résultat d'une interaction complexe entre ces facteurs.

10 Une étude multi-sites menée de juin 1997 à mars 1998 dans quatre villes africaines Cotonou, Yaoundé, Ndola et Kisumu - a montré que plusieurs déterminants s'avéraient décisifs dans une prévalence élevée: âge du partenaire et précocité des relations sexuelles associée à une plus forte prévalence ; non-utilisation du préservatif ; maladies sexuellement transmissibles ulcératives; non-circoncision associée à une plus forte prévalence (Buvé et al., 2001).

11 Outre l'effet des deux groupes de déterminants exposés, d'autres phénomènes - le sousdéveloppement, la pauvreté individuelle et celle des États, les précarités et marginalités (économique, socio-culturelle), les inégalités de genre constituent un terreau de vulnérabilités. En situation de survie au quotidien, l'individu peut être peu sensible à des mesures de prévention qui signifient se projeter dans l'avenir. Le manque de volonté des pouvoirs étatiques, les déficiences des systèmes de santé ont été des obstacles à la prévention des MST et au dépistage de la séropositivité. L'ignorance et les insuffisances de la prévention sont susceptibles de désengager l'individu de toute responsabilité.

12 Le schéma suivant expose les différents groupes de déterminants (biologiques, comportementaux, sociaux et écologiques) qui interviennent dans l'exposition, la transmission et la diffusion du VIH. 
La transmission et la diffusion du VIH : déterminants biologiques, comportementaux, sociaux

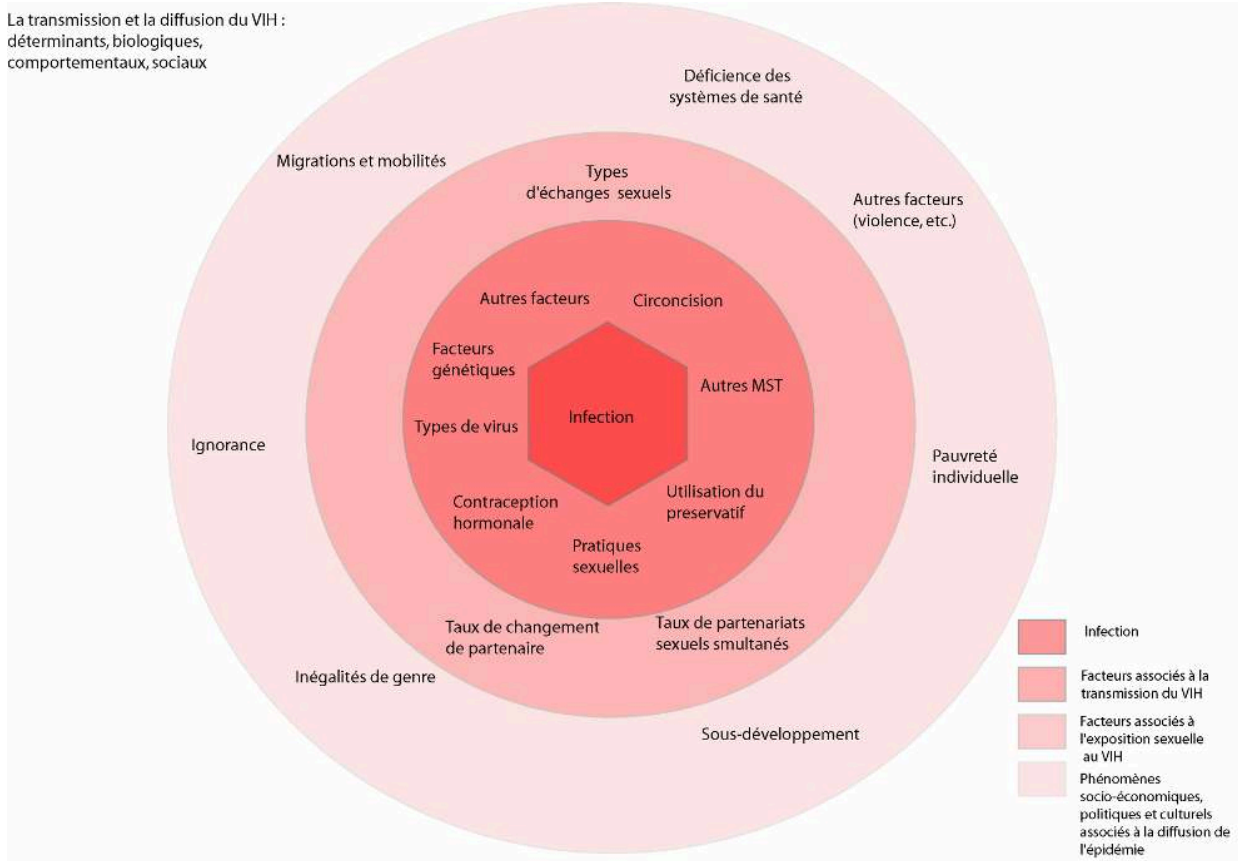

Source : M. Miralles, 2007 des inégalités sociales et disparités spatiales face à l'infection. En effet, les données de séroprévalence indiquent que la prévalence de l'infection à VIH est plus élevée parmi les populations urbaines. Dans le même temps, le KDHS 2003 indique qu'il n'y a pas de lien entre prévalence au VIH et pauvreté alors que plusieurs recherches ont souligné la plus grande vulnérabilité à l'infection à VIH des populations vivant dans les "slums ». Dans l'affirmative, comment expliquer la plus forte exposition à l'infection à VIH des populations vivant dans les slums?

Après avoir évoqué les spécificités des travaux géographiques portant sur l'épidémie (I), puis dressé un tableau de son évolution au Kenya (II), nous brosserons une synthèse des facteurs épidémiologiques, sociaux et géographiques associés à la transmission et la diffusion du VIH au sein de la population (III). In fine, nous établirons un tour d'horizon des facteurs de risques connus et des principales hypothèses sur les composantes de l'environnement des «slums» de Nairobi favoriser la vulnérabilité à l'infection à VIH (IV). 


\section{La contribution de la géographie à la compréhension de l'épidémie à VIH-Sida : une approche marquée par des liens avec l'épidémiologie et les sciences sociales}

17 La géographie humaine, et plus singulièrement la géographie de la santé, ont apporté leur contribution à l'étude de l'épidémie à VIH dans le sillage de l'épidémiologie et des sciences sociales.

18 Les études géographiques se sont plus naturellement portées vers les phénomènes associés à l'épidémie à VIH avec une dimension socio-spatiale : relation entre mobilité géographique, migrations et prévalence; urbanisation et prévalence; analyse des variations régionales et périodisation de l'épidémie, etc.

19 Dans l'éventail des publications, les analyses du rôle du facteur migratoire dans la diffusion de l'épidémie ont occupé une place prépondérante.

Concernant l'effet de la mobilité humaine sur la diffusion du sida, une première catégorie de travaux a proposé des schémas d'analyse qui à la hiérarchie des villes et axes de communication, associent des pôles, carrefours, couloirs de diffusion de l'épidémie (Amat-Roze, 1989 et 1993; Gould, 1993). D'autres recherches se sont efforcées de comprendre comment les relations entre migrations et sida se modifiaient au long du processus épidémique, selon les formes de mobilités et les catégories de sujets impliqués. En Afrique de l'Ouest, Gérard Rémy a ainsi esquissé une analyse du rôle joué par le facteur migratoire dans la diffusion de l'épidémie en mettant en avant le rôle des groupes noyaux - prostituées - puis celui des groupes mobiles complémentaires - routiers et marchands -, et enfin des migrants de travail pour la propagation de l'épidémie à VIH (Rémy, 1993 ; 2002).

21 De façon générale, les recherches géographiques sur le rôle de la mobilité sont avant tout préoccupées par les aspects épidémiologiques du sida. Dans cette perspective, les études mettent en avant que le $\mathrm{VIH}$, comme d'autres infections, se transmet de personne en personne et circule dans les directions et aux rythmes des déplacements humains. Un nombre important de recherches géographiques sont venues vérifier pour l'Afrique Subsaharienne, ces associations claires entre la dynamique spatiale de l'épidémie, la migration de travail et les migrations forcées (Prothero, 1994 et 1996 ; Amat-Roze, op. cit), l'urbanisation (Lydié et al., 2000) et les axes routiers. Cette lecture présente l'intérêt d'informer sur la dynamique de l'épidémie. Mais une critique majeure qui lui a été faite se situe dans la manière dont elle a posé la relation entre migration et sida de manière mécanique, sans références explicites aux modes de transmission.

22 Notons ici que l'approche géographique du rôle de la mobilité humaine se singularise des recherches menées par les socio- démographes et les anthropologues qui mettent l'accent sur les situations sociales partagées par de nombreux travailleurs migrants (Decosas et al., 1995) susceptibles de les exposer à différents facteurs de risques associés à l'infection à VIH.

23 Cette polarisation des recherches géographiques sur la dynamique de diffusion de l'épidémie et les liens clairs établis avec l'épidémiologie se traduisent également par l'élaboration de cartographies de la magnitude de l'épidémie, de cartes synthétiques de la situation, ou encore l'établissement de corrélations entre le niveau de prévalence et des données socio-économiques, démographiques pour l'étude des variations entre 
aires rurales et urbaines, régions et pays. L'explication des variations régionales de l'épidémie se situe dans une combinaison des approches biomédicale et des sciences sociales et humaines. L'intérêt d'une approche géographique en aval de ces différentes recherches est alors d'offrir une vision spatialisée, diachronique ou synchronique de la dynamique de diffusion de l'épidémie mais aussi d'identifier le rôle de phénomènes tels que la migration.

L'apport de la géographie à la recherche sur l'épidémie à VIH s'est aussi singularisé par l'utilisation de différentes notions empruntées à son corpus conceptuel: territoires (Lalou et al. 2006; Lydié, 2001); espaces de vulnérabilité (Williams et al. 2002); environnement (concept commun à de nombreuses disciplines des sciences sociales). Ces notions ont été utilisées par des démographes ou des spécialistes de santé publique pour définir une toile de fond ou un cadre pour l'interprétation des différenciations géographiques de l'épidémie et des risques qui lui sont associés.

En effet, la dimension géographique de recherches réalisées en Afrique Subsaharienne par des spécialistes d'autres disciplines est patente. En République Démocratique du Congo, les disparités géographiques de séroprévalence observées entre 11 des plus grandes villes du pays ont donné lieu à une vaste enquête socio-démographique et anthropologique dont l'objectif a été d'analyser ces disparités, en mettant en lumière différents environnements à risques. Cette recherche a posé comme hypothèse que les collectivités urbaines avaient été traversées par les crises politiques et économiques de ces dernières décennies ni au même moment ni avec la même intensité. Des environnements à risques différents s'étaient profilés entre les villes. Ils pouvaient être déclinés en trois composantes: les guerres civiles et leurs cortèges de violence, les migrations forcées pour raisons économiques et la montée en puissance du religieux. La multiplicité de ces environnements avait agi sur le rythme différencié de la propagation de l'épidémie dans le territoire.

26 Par le choix des échelles d'analyse - l'échelle territoriale -, la sémantique de l'étude territoire, environnements à risques -, les phénomènes pris en compte - disparités, migrations, l'usage d'une typologie de villes comme cadre d'interprétation - et le recours à un appareil cartographique pour illustrer des différenciations entre comportements sexuels selon des villes, cette recherche met en lumière des liens avec une approche géographique.

27 Cette dimension géographique dans l'analyse de la diffusion de l'épidémie est aussi manifeste dans une étude réalisée en Afrique du Sud « Spaces of Vulnerability: Migration and HIV/AIDS in South Africa " (Williams, op. cit). Cette étude collective issue d'une thèse en santé publique s'intéresse aux liens entre la mobilité humaine et l'épidémie à VIH en Afrique du Sud. L'état des lieux des connaissances relatives à cette problématique «migrations-SIDA» s'appuie explicitement sur une géographie du fait migratoire en Afrique du Sud et sur une macro-géographie du VIH-SIDA qui dresse un tableau de la situation épidémiologique du pays selon les aires rurales et urbaines, les régions. In fine, cette étude varie les échelles d'analyse pour analyser les relations entre mobilité et VIH : à Carltonville - plus vaste mine d'or au monde -; en milieu rural dans le district de Hlabisa ; et sur un axe routier entre Durban et Johannesburg. L'ensemble des analyses effectuées s'appuie, là encore, sur un appareil cartographique varié.

28 Au total, la géographie offre un certain nombre d'outils pour analyser la dynamique spatiale de l'infection grâce aux apports de l'épidémiologie. Cette discipline présente également la possibilité de dresser des typologies de lieux en fonction de l'exposition et 
de la vulnérabilité au VIH des populations ou encore des niveaux de prévalence observés. Dans cette perspective de recherche, la géographie affirme ses complémentarités avec les études sociodémographiques, anthropologiques.

La contribution de ces dernières permet de mettre en lumière, en un lieu donné, les environnements sociaux qui peuvent sous-tendre l'exposition ou la vulnérabilité à l'infection à VIH. Le but est de repérer à travers une étude des conditions de vie des gens (un groupe, une "communauté", la population générale) les dynamiques susceptibles de créer les situations de vulnérabilité sociale et individuelle. Les enquêtes socio-anthropologiques se profilent alors comme un outil efficace pour dévoiler les mécanismes qui font d'un bidonville ou d'une ville un espace porteur de vulnérabilités ou un environnement favorisant l'exposition aux risques. La prise en compte des environnements physiques - le bidonville, la ville - et sociaux lie alors la géographie à l'écologie humaine.

\section{Situation épidémiologique : les disparités de l'épidémie à VIH-Sida au Kenya}

30 La distribution de l'épidémie à VIH/sida au Kenya est marquée par des disparités importantes suivant les populations, les régions, les aires urbaines et rurales. Cette hétérogénéité de l'épidémie s'expliquerait par l'effet singulier ou la conjonction de facteurs de différentes natures: bio-médicaux, comportementaux et socioéconomiques. De façon générale, l'épidémie semble avoir marqué un léger recul entre 2003 et 2005 : les estimations de prévalence dans la population adulte sont en effet passées de $6,8 \%$ à $6,1 \%$. (UNAIDS, 2006).

\subsection{Des disparités géographiques et de genre}

31 Deux sources d'estimation distinctes sur l'évolution de l'épidémie de VIH convergent sur les disparités géographiques et de genre qui caractérisent sa diffusion. Chacune de ces sources comporte des biais, mais elles livrent une vue d'ensemble de la distribution de l'épidémie à VIH complémentaire aux enquêtes épidémiologiques ponctuelles. À l'échelle du Kenya, ces deux sources sont:

- Les données de séroprévalence du réseau sentinelle, réestimées par l'OMS et l'ONUSIDA ${ }^{1}$. Elles prennent en compte des femmes enceintes et des professionnelles du sexe.

- Les données de séroprévalence du Kenya Demographic and Health Survey 2003.

Le réseau sentinelle a été mis en place à la fin des années 1980 par le gouvernement kényan. Il s'agit d'un système national de surveillance de l'épidémie fondé sur des enquêtes longitudinales portant sur des populations de femmes enceintes et des patients infectés par des maladies sexuellement transmissibles (MST) dépistés dans 42 sites sentinelles. Ces sites localisés dans des aires rurales et urbaines sont censés représenter l'hétérogénéité du peuplement du Kenya (UNAIDS, 2005). Actuellement, un quart des sites du réseau sentinelle se trouverait dans des aires « rurales» regroupant moins de 10000 habitants $^{2}$.

Les données sont collectées durant trois mois chaque année via le dépistage de femmes enceintes enregistrant leurs premières visites en maternité et de patients présentant des MST. À l'instar des autres pays abritant des sites sentinelles, ces données sont 
supposées livrer un panorama général de la prévalence au VIH parmi la classe d'âge 15-49 ans considérée comme la plus active sexuellement (UNAIDS, 2005).

Toutefois, cet indicateur souffre de plusieurs limites. Les taux de prévalence du VIH ne donnent pas un décompte exact du nombre de personnes infectées par le VIH (Williams et al. 2002). Ils sont utilisés pour livrer une estimation partielle de l'évolution de l'épidémie, de ses tendances temporelles et de ses variations géographiques.

En outre, le taux de prévalence au sein du groupe des femmes enceintes n'offre pas une appréciation juste de la diffusion du VIH au sein des populations féminine et masculine dans leur ensemble. En effet, les données du réseau sentinelle sont partielles. Ainsi, elles ne prennent pas en compte les femmes qui ne vont pas accoucher dans les maternités du réseau. De plus, deux autres limites doivent être soulignées concernant la séroprévalence de la population féminine (Williams et al., 2002): d'une part, les données de séroprévalence du réseau sentinelle pourraient surestimer la prévalence de l'infection parmi les jeunes femmes, car les femmes enceintes pourraient être plus sexuellement actives que les sujets qui ne le sont pas. D'autre part, concernant les femmes plus âgées, la surveillance sentinelle pourrait sous-estimer leur séroprévalence car celles-ci pourraient être plus exposées aux MST qui favorisent l'infection à VIH. In fine, le VIH est associé à une réduction de la fécondité parce que la connaissance du statut (par la femme et/ou le conjoint) est susceptible d'influencer les pratiques reproductives (Desgrées du Lou, 2006).

S'agissant d'étudier la population masculine, la prévalence féminine ne constitue pas un bon indicateur car les enquêtes épidémiologiques ponctuelles soulignent des taux et risques d'infection moins importants parmi les sujets masculins. (KDHS, 2003).

La distribution de la prévalence du VIH dans les sites du réseau sentinelle

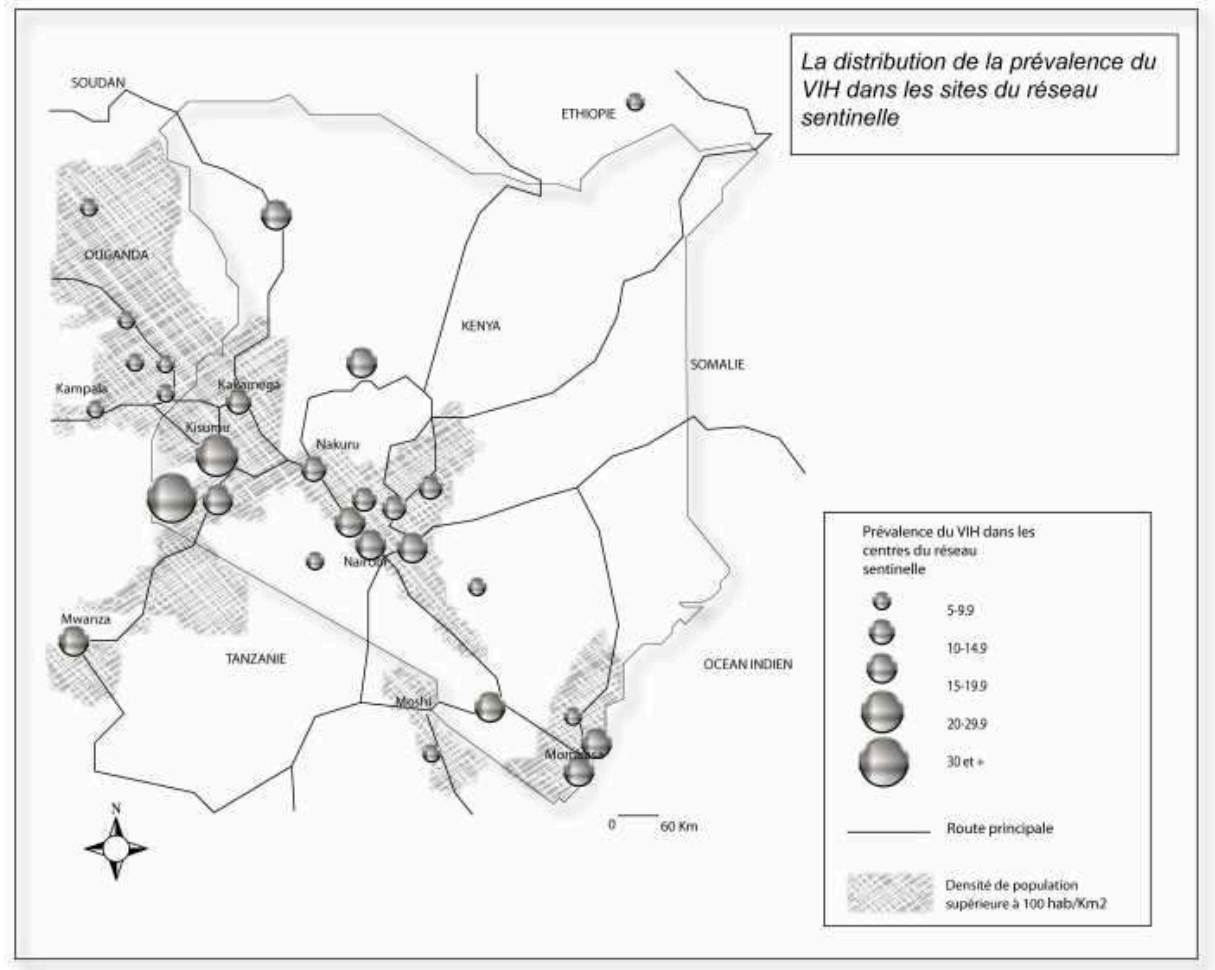

Cartographie : M. Miralles 

réseau sentinelle, la prévalence du VIH, dans le groupe des femmes enceintes, s'élevait en en 2004 à $10 \%$ dans les aires urbaines contre $6 \%$ dans les aires rurales. En milieu urbain et rural, il semble que l'épidémie ait amorcé un léger recul au sein de la population depuis 2002 . prévalences les plus faibles étaient relevées dans le nord du Kenya à Garissa (1\%).

Dans la capitale, la prévalence du VIH parmi les femmes enceintes semble assez disparate suivant les quartiers : en 2004, elle s'élevait à $5 \%$ dans des maternités du district de Dagoretti contre $15 \%$ dans les quartiers de Kariobangi et de Kibera qui abritent de vastes bidonvilles (UNAIDS, 2006 ; MSF B, 2006). Ces donnes ne sauraient toutefois être considérées comme représentatives de la prévalence au VIH de la population de ces quartiers. À l'échelle de la capitale, on peut néanmoins observer une baisse générale de la prévalence depuis les années 1990 au sein de la population de Nairobi.

40 À Mombasa, cet indicateur se situait dans une fourchette échelonnée entre $16,3 \%$ en 1998 et $10 \%$ en 2004.

41 À Kisumu, la prévalence a été fluctuante et a connu son point d'acmé en 2000 avec près de $35 \%$ de séropositives parmi les femmes dépistées. Selon, l'étude multisites à laquelle Kisumu était intégrée (Buvé et al., 2001), les jeunes femmes étaient plus susceptibles d'être infectées que les hommes du même âge. Parmi, les femmes âgées de 15 à 29 ans, la prévalence était estimée à 33,7 \% alors que pour les hommes, elle s'élevait à 14,3\%.

\begin{tabular}{|l|l|l|l|l|l|}
\hline $\begin{array}{l}\text { Estimation fin } \\
\mathbf{2 0 0 5}\end{array}$ & $\begin{array}{l}\text { Adultes } \\
\mathbf{( 1 5 + )}\end{array}$ & $\begin{array}{l}\text { Enfants } \\
\mathbf{( 0 - 1 4 )}\end{array}$ & $\begin{array}{l}\text { Femmes } \\
\mathbf{( 1 5 + )}\end{array}$ & $\begin{array}{l}\text { Nombre de décès } \\
\text { en 2005 }\end{array}$ & $\begin{array}{l}\text { Nombre } \\
\text { d'orphelins }\end{array}$ \\
\hline $\begin{array}{l}\text { Estimation } \\
\text { basse }\end{array}$ & 990000 & 55000 & 640000 & 110000 & 890000 \\
\hline $\begin{array}{l}\text { Estimation } \\
\text { haute }\end{array}$ & 1300000 & 290000 & 840000 & 170000 & 1300000 \\
\hline
\end{tabular}

Le Kenya Demographic and Health Survey 2003 (KDHS, 2003), basée sur des enquêtes dont le taux de réponse se situait autour de $63 \%$ en milieu urbain ( $52,4 \%$ à Nairobi) et $79,3 \%$ en milieu rural, livre une estimation de la prévalence nationale de $6,7 \%$ avec des différences entre urbains $(10 \%)$ et ruraux $(5,6 \%)$ mais aussi entre hommes $(4,5 \%)$ et femmes $(8,7 \%)$. Le ratio homme-femme est plus haut que dans la plupart des pays africains.

43 En effet, les informations du KDHS indiquent que $3 \%$ des femmes âgées de 15 à 19 ans étaient séropositives au moment de l'enquête contre $0,25 \%$ des hommes de la même classe d'âge. La prévalence s'élevait à $9 \%$ parmi les femmes âgées de 20 à 24 ans contre $2 \%$ chez les hommes. Dans la population féminine, le pic de prévalence s'élève à $13 \%$ pour la classe d'âge 25-29 ans alors que pour les hommes, elle atteint son niveau le plus élevé dans la classe d'âge 40 / 44 ans.

En termes de disparités géographiques, la province de Nyanza qui compterait $15 \%$ de personnes séropositives est talonnée par Nairobi avec $10 \%$ de sujets dépistés séropositifs. Les populations des autres provinces connaissent des prévalences entre 4 
et $6 \%$. Dans la North Eastern Province, aucun répondant n'a été testé positif. Selon le KDHS, les deux groupes ethniques avec les prévalences les plus importantes sont les Luo et les Taita : plus d'une Luo sur quatre et $18 \%$ des hommes seraient séropositifs. La prévalence serait également plus importante chez les chrétiens comparés aux musulmans (9\% contre $3 \%$ ).

La comparaison intranationale des séroprévalences montre une grande diversité de situations après deux décennies épidémiques: différences de séroprévalence, différences selon les milieux, agglomérations urbaines et bourgs ruraux.

\section{L'épidémie à VIH au Kenya : des déterminants biologiques aux facteurs sociaux et géographiques}

Les déterminants biologiques et sociaux qui peuvent être associés aux disparités de l'épidémie à VIH au Kenya sont multiples. La synthèse des principales études épidémiologiques ou relevant des sciences sociales esquissée ici met en évidence que le canevas de déterminants associés à l'exposition et à la transmission du virus est constitué des différents facteurs identifiés en Afrique Subsaharienne : non-circoncision, MST, âge au premier rapport, comportement sexuel, pauvreté, etc. ${ }^{3}$ La propagation aurait quant à elle été favorisée par les dynamiques migratoires et mobilités géographiques...

\subsection{Les déterminants biomédicaux et comportementaux de l'épidémie à VIH au Kenya}

Les données à notre disposition indiquent que le mode prédominant de transmission du VIH au Kenya, à l'instar de l'Afrique Subsaharienne, est la relation sexuelle entre un homme et une femme.

Plusieurs facteurs sont susceptibles d'accroître le risque de transmission au cours d'une relation hétérosexuelle. D'un point de vue épidémiologique, ces déterminants relèvent de deux groupes de facteurs distincts associés, soit à l'exposition sexuelle, soit à la transmission du virus.

\subsubsection{La diffusion de l'épidémie à VIH : modes de transmission et déterminants}

\section{Les modes de transmission prédominants}

Les relations hétérosexuelles sont le principal mode de transmission du VIH au Kenya. Elles compteraient pour $75 \%$ des infections au VIH (KDHS, 2003). Ce mode de transmission devance la transmission périnatale qui peut se produire durant la grossesse, au moment de la naissance ou durant l'allaitement. On estime que 30 à $40 \%$ des enfants nés de femmes séropositives seront à leur tour contaminés (KDHS, op. cit). En 2005, selon le rapport annuel de l'UNAIDS, on estime qu'entre 410000 et 666000 enfants auraient été contaminées par transmission périnatale depuis les débuts de l'épidémie. 


\section{Les déterminants associés à l'exposition au VIH : le comportement sexuel} qu'un taux élevé de partenaires sexuels était associé au niveau individuel à un risque accru d'infection. Une étude réalisée au Kenya a ainsi montré que les femmes avec deux partenaires avaient un risque d'exposition au VIH - 2,5 - supérieur à celui des femmes qui n'avaient qu'un partenaire. (Hunter, 1994). En outre, de nombreuses études ont identifié comme facteur de risques les relations sexuelles marchandes entre femmes et hommes.

51 Les résultats des simulations faites à l'aide de modèles mathématiques suggèrent que certains types de comportements sexuels provoquent une explosion de l'épidémie. C'est le cas notamment quand les hommes ont des relations sexuelles avec un petit groupe de femmes très actives sexuellement, comme les travailleuses de l'industrie du sexe et quelques relations avec des femmes qui sont peu actives sexuellement (Anderson, 1991). De même si de nombreuses personnes appartenant à différentes classes d'âges ont des relations sexuelles entre elles, par exemple dans le cas de femmes jeunes et d'hommes bien plus âgés qu'elles, le taux de propagation du VIH dans la population se trouve démultiplié. Plus récemment, il a été suggéré que pour le même taux de multiplicité de partenaires, des relations concurrentes ont plus d'impact sur la propagation du virus que des relations sexuelles successives. Ce faisant, une tendance aux relations concurrentes augmente la probabilité d'une explosion de l'épidémie dans la population (Morris, 1997).

À l'instar de l'Afrique Subsaharienne, il apparaît au Kenya que les comportements à risque sont aussi liés à l'âge et au genre. traduiraient par un risque significatif d'infection (KDHS, 2003 ; Hargreaves et al., 2002). Plus généralement, les jeunes femmes âgées de 15 à 24 ans dont le premier rapport sexuel avait eu lieu avec des hommes âgés d'au moins dix ans de plus qu'elles avaient une plus forte prévalence $(10 \%)$ comparées à celles dont le premier partenaire était plus jeune $(8 \%)$. Les hommes ayant connu des relations sexuelles au mitan de l'adolescence n'auraient pas la même exposition au risque.

Dans un autre ordre d'idée, les femmes ayant des partenaires, hors mariage et ne résidant pas avec eux, ont une prévalence de $17 \%$ comparées aux autres (8\%). En outre, le multipartenariat dans la population féminine serait associé à une prévalence plus élevée alors que le lien ne serait pas significatif pour les hommes (KDHS, 2003). Les relations sexuelles accompagnées d'une contrepartie matérielle - monétaire ou non pour les femmes comme pour les hommes sont associées à une prévalence élevée mais dont la significativité est patente seulement pour les hommes (11\% contre $10 \%$ pour les femmes, $8 \%$ contre $4 \%$ pour les hommes).

Si l'on élargit l'éventail des déterminants aux co-facteurs de risque (i.e. aux facteurs qui n'ont pas d'effet direct sur l'exposition ou la transmission du VIH), la prise d'alcool est aussi associée à une forte prévalence (Hargreaves et al. 2002) : $19 \%$ des femmes et $6 \%$ des hommes ayant déjà pris de l'alcool étaient infectées contre respectivement $9 \%$ et $4 \%$ pour les individus ne l'ayant pas mentionné. 


\section{Les facteurs influençant la transmission durant l'acte sexuel}

Le préservatif est considéré comme l'un des moyens les plus efficaces pour contenir la transmission et la propagation du VIH. À l'échelle de l'Afrique Subsaharienne, une méta-analyse sur des couples séro-différents a établi que l'utilisation du préservatif peut réduire jusqu'à $69 \%$ le risque de transmission du VIH (Grosskurt et al., 1995). En outre, tout au long des années 1980 et 1990 - période durant laquelle l'épidémie a atteint son point d'acmé dans la majorité des pays africains -, les préservatifs furent utilisés par moins de $1 \%$ des couples mariés.

Dans le cas du Kenya, il est clair que les relations sexuelles non-protégées constituent un facteur de risque important.

Cependant, il semble qu'un nombre important de Kényans, ces dernières années, ont adopté des comportements sexuels plus sûrs. C'est étonnamment chez les femmes que l'utilisation du préservatif avec des partenaires occasionnels a le plus augmenté : en 2003, près de $24 \%(23,9 \%)$ d'entre elles déclaraient avoir utilisé un préservatif lors de leur dernier rapport occasionnel, contre $15 \%$ cinq ans plus tôt. (KDHS, 2003). Ce recours plus fréquent à l'usage du préservatif coïncide avec la baisse de la prévalence observée à l'échelle du pays, depuis quelques années.

Les relations sexuelles non-protégées exposent aux maladies sexuellement transmissibles (MST) qui favorisent elles aussi la transmission du VIH.

En effet, il est maintenant établi que des MST comme les infections sexuellement transmissibles ulcérantes, l'herpès simplex II (HSV2), la syphilis, la trichomonase, les MST non-ulcérantes (la gonorrhée et/ou infection à Chlamydia) facilitent la transmission de l'infection au cours de l'acte sexuel et peuvent jouer un rôle clé dans la dynamique de l'épidémie.

Plusieurs études en Afrique Subsaharienne ont établi que les infections sexuellement transmissibles augmentent la réceptivité au VIH des individus non infectés et le pouvoir infectant des individus porteurs du VIH. Après avoir été mise en évidence par des études épidémiologiques sur la séro-conversion du VIH, cette relation entre MST dites classiques et VIH a été confirmée par une étude comparative ayant montré, à la suite d'une amélioration dans la détection des cas et le traitement des IST une réduction de $40 \%$ de l'incidence du VIH dans la population générale de Mwanza en Tanzanie (Grosskurt et al., 1995)

La prévalence des MST peut être très hétérogène dans la population.

Il existe plusieurs explications possibles de la variation du taux de prévalence des MST parmi lesquelles :

- les différences dans le comportement sexuel (taux de partenaires et type de mixité);

- des différences dans l'accès et le recours à des traitements efficaces ;

- des variations dans la prévalence d'autres facteurs pouvant augmenter le risque de certaines IST tels que la non-circoncision des hommes et l'hygiène génitale...

À l'instar de l'Afrique Subsaharienne, sans en dresser un inventaire exhaustif, plusieurs enquêtes réalisées au Kenya dans différentes populations démontrent le lien prépondérant entre une forte prévalence au VIH et les MST. En effet, l'étude multisite réalisée à Kisumu-Ndola-Yaoundé et Cotonou (Buvé et al., 2001) associe, conjointement à d'autres facteurs, la forte prévalence au VIH relevée dans la population féminine avec 
l'herpès génital (HSV 2) et la trichomonase. Ces facteurs, conclut l'étude, auraient eu un rôle plus significatif que les facteurs comportementaux. groupes ethniques au sein desquels la circoncision est une norme. Les Luo compteraient selon les estimations du KDHS (2003) $17 \%$ d'individus circoncis. Rappelons qu'il s'agit du groupe ethnique au sein duquel la prévalence est la plus élevée.

71 Dans le canevas des facteurs facilitant la transmission du virus, il convient également d'évoquer le rôle qu'est susceptible de jouer le type de virus. trois types de VIH-1 (groupes $\mathrm{M}, \mathrm{N}$ et $\mathrm{O}$ ). L'ensemble a été repéré en Afrique Subsaharienne.

des preuves épidémiologiques, la transmissibilité du VIH-1 est plus élevée que celle du VIH-2. Mais aucune preuve convaincante n'a été établie au sujet de l'existence de différences majeures dans la transmissibilité et la pathogénèse des diverses souches de VIH-1, différences qui pourraient expliquer les variations entre populations (Mac Cutchan, 2000).

En outre, jusqu'à ce jour, aucun facteur génétique n'a été trouvé qui conférerait une résistance à l'infection par le VIH au niveau d'une population et qui serait suffisamment prévalent dans une population pour avoir un impact sur le taux de propagation.

76 Le VIH-1, dont il a été établi que la transmissibilité était plus forte que celle du VIH-2, est considéré comme prédominant au Kenya.

Les Cahiers d'Afrique de l'Est / The East African Review, 39 | 2008 
On pourrait s'interroger sur d'autres facteurs susceptibles de favoriser la transmission $\mathrm{du}$ VIH. Cependant, nous ne pouvons évoquer leur impact sur la diffusion du VIH au Kenya faute d'études sur leur rôle.

Ainsi, en Afrique Subsaharienne, des études sur les couples sérodiscordants ont établi qu'une relation sexuelle durant la période de règle allait de pair avec un risque accru d'infection par le VIH chez le partenaire masculin de la femme contaminée, mais non chez le partenaire féminin de l'homme contaminé.

De la même façon, la pratique du dry sex - relation sexuelle pour laquelle la femme garde son vagin sec et non dilaté - s'accompagnerait d'un risque accru de transmission en raison des lésions qu'elle provoque. (Brown, 1993)

De façon plus marginale, il a été avancé que pour les femmes le recours à une contraception hormonale était un facteur de risque d'infection. Cependant, des doutes demeurent à ce sujet. Il est peu probable qu'il s'agisse d'un facteur déterminant pour expliquer la propagation au Kenya et plus largement en Afrique Subsaharienne (Wang, 1999)

81 De nombreux facteurs liés à l'exposition sexuelle ou encore à la transmission du virus expliquent la diffusion hétérogène de l'épidémie à VIH au Kenya. L'étude multi-sites réalisée à Kisumu, Ndola, Yaoundé et Cotonou permet de cerner le rôle des différents facteurs.

\section{L'étude multisites : une approche écologique (avec le groupe comme référence) combinée à une analyse individuelle de type épidémiologique}

2 Les résultats de l'étude multisites réalisée à Kisumu, Ndola, Yaoundé, Cotonou (Buvé et al., op. cit.) permettent de prendre la mesure du rôle joué par les différents facteurs associés à des comportements à risques ou susceptibles de favoriser la transmission du VIH. Les résultats de cette enquête ont déjà été évoqués en filigrane des sous-parties précédentes.

3 Une synthèse de cette étude présente néanmoins l'intérêt d'esquisser une hiérarchie des différents déterminants liés au taux de prévalence élevé au sein de la population de Kisumu. Le protocole d'enquête conjuguait une approche écologique prenant le groupe comme unité statistique de référence à une enquête épidémiologique s'appuyant sur des analyses individuelles permettant de dépasser le simple constat de corrélation en remontant à la cause.

Dans deux des quatre sites d'étude - Cotonou et Yaoundé -, la prévalence de l'infection était faible alors qu'à Kisumu et Ndola, la prévalence dépassait les $20 \%$.

Le rapport entre la prévalence du VIH chez les hommes et la prévalence chez les femmes était de 1.5 à Kisumu. Dans la tranche d'âges 15-19 ans, la prévalence chez les femmes était six fois supérieure à celle constatée chez les hommes ( $23 \%$ pour les femmes, $3,5 \%$ pour les hommes)

Il est ressorti de l'analyse que les comportements sexuels tels que décrits par les jeunes femmes ne pouvaient expliquer ces différences de prévalence entre les populations des quatre villes. En revanche, les facteurs tels que l'infection d'Herpès génital pouvaient avoir joué un rôle important.

La non-circoncision a été identifiée comme un facteur significatif parmi la population masculine en termes de corrélation avec une forte prévalence. 
88 De plus, les MST ulcéreuses et notamment l'herpès génital et la trichomonase avaient une prévalence plus importante à Kisumu et Ndola. À Kisumu, $37 \%$ des hommes souffraient d'herpès simplex virus type 2 et $69 \%$ des femmes (contre 29 et $31 \%$ respectivement à Yaoundé).

$89 \mathrm{Au}$ total, cette étude a mis en avant l'importance de facteurs ne relevant pas principalement du comportement sexuel sur l'ampleur prise par l'épidémie à VIH à Kisumu et plus largement dans les trois autres sites d'enquête.

$90 \mathrm{Au}$ total, sur le plan épidémiologique, la propagation du VIH au sein de la population kényane est le produit d'un faisceau complexe d'éléments ayant trait au comportement sexuel et à des co-facteurs qui accélèrent la transmission du VIH. Notons que la diffusion hétérogène de l'épidémie semble en partie liée à la non-circoncision et aux MST. Parmi les autres facteurs de risque en cause, on peut relever la précocité des relations sexuelles chez les jeunes femmes, les relations sexuelles non-protégées. Cependant, les comportements et pratiques sexuelles sont aussi susceptibles d'être façonnés par les milieux sociaux, économiques et culturels.

\subsection{L'effet des déterminants socio-économiques et de la pauvreté}

91 Les relations entre prévalence du VIH et condition socio-économique des individus ont été fréquemment explorées (Farmer, 1996).

92 Pauvretés étatique et individuelle favorisent l'exposition au risque de transmission et limitent la riposte. Cependant, les territoires les plus affectés ne sont pas forcément les plus pauvres. La dynamique infectieuse soulève plus d'interrogations qu'elle n'apporte de réponses. À côté de puissants dénominateurs communs observables sous toutes les latitudes, de l'autre des données locales ou régionales qui modifient ou atténuent ces dénominateurs communs notamment sur les liens entre pauvreté et risque associé au VIH-SIDA.

$93 \mathrm{Au}$ Kenya, des enquêtes anthropologiques et épidémiologiques réalisées à Kisumu attestent de cette relation notamment chez les jeunes femmes (Tyndall, 2000). Une enquête épidémiologique réalisée dans cette même ville (Hargreaves et al., 2002) auprès d'individus de statuts socio-économiques différents a montré que la prévalence au VIH était plus élevée au sein d'un groupe de femmes avec de très bas revenus appartenant à la classe d'âge 15-24 ans. Au sein des autres groupes, le risque était moins élevé même si les taux enregistrés étaient plus importants que la moyenne nationale. Dans ce groupe de jeunes femmes, la prévalence de l'herpès génital (HSV 2) était aussi marquée. En outre, elles se différenciaient également des autres groupes dépistés par des relations sexuelles précoces et par un faible usage du préservatif. Dans l'ensemble de la population dépistée au cours de cette enquête (indiquant une prévalence au VIH de l'ordre de 19,8\% pour les hommes et 30,2\% pour les femmes), les autres facteurs de risques associés au sida mis en exergue se situaient dans la prise d'alcool et aussi l'absence de circoncision chez les hommes.

94 À la différence des résultats de cette enquête réalisée à Kisumu, le KDHS 2003 indiquait que les classes sociales, caractérisées par des niveaux de revenus élevés, étaient singularisées par les plus forts taux de prévalence $(12,2 \%)$ contre $3,9 \%$ pour les populations avec les plus bas revenus. Cette différence coïncide avec le fait que la plupart des "pauvres" - en termes de répartition géographique de la population résident en milieu rural où la prévalence est plus faible. Cette situation pourrait niveler 
vers le bas la prévalence relevée dans la population aisée fortement représentée dans les grandes villes, et notamment la capitale, où en 1995, les individus vivant en dessous du seuil de pauvreté constituaient $60 \%$ de la population (APHRC, 2002).

\subsection{Les variables de propagation de l'épidémie à VIH au Kenya : les dynamiques migratoires et mobilités géographiques}

Les échanges migratoires conjugués au processus d'urbanisation semblent lies à la distribution hétérogène de l'épidémie à VIH.

Une étude spatio-temporelle de la diffusion de l'épidémie a été réalisée dans les années 1990 (Ouma, 1997). Cette étude qui s'inscrit dans une double perspective géographique et écologique a mis en lumière que la diffusion de l'épidémie était associée à la trame urbaine, aux densités humaines et à la proximité des principaux axes routiers.

Le réseau viaire a également été un critère déterminant dans l'étude réalisée par un spécialiste de Santé Publique Canadien. En s'appuyant sur des systèmes d'information géographiques et des photos aériennes, l'auteur a localisé sur le principal corridor routier du Kenya, qui s'étire de l'Ouganda à Mombasa, les carrefours de la prostitution fréquentés par les routiers (Morris, 2004). Puis il a réalisé une enquête sur les principaux facteurs de risques parmi les populations fréquentant ces différents lieux avant de livrer une estimation du nombre d'infections à venir.

Cette étude concluait, qu'il pouvait y avoir 2235 nouvelles infections en 2005 sur la route Mombasa-Busia, soit $3,7 \%$ des nouvelles infections susceptibles d'être décomptées au Kenya dans l'année suivant l'enquête.

L'effet route se traduit par des services types liés à la vie de relation: marché, petit commerce, bar, gîtes d'étape, station-service pour automobiles... dont la prospérité repose sur des hommes en déplacement. Résider en ville ou dans des agglomérations à caractère urbain est une caractéristique associée à une séroprévalence élevée observée par de nombreuses études. Ainsi on constate, d'une façon, qui est loin d'être exceptionnelle, la relation entre situation de frontière et haute séroprévalence comme l'indique la situation épidémiologique dans la ville de Busia. Les localisations nous enseignent qu'aucun lieu ne peut être considéré à l'abri de l'importation du virus mais aussi que certains paraissent privilégiés (Amat-Roze, 2003)

Les disparités face au VIH observées entre aires rurales et urbaines se juxtaposent avec l'urbanisation explosive que connaissent les villes du Kenya depuis 40 ans. Or, cette urbanisation explosive a été pour une large part alimentée par des migrations ruralesurbaines qui constituent un phénomène structurant de la société kényane depuis l'indépendance.

À l'échelle du Kenya, la migration se profile comme un facteur favorisant les comportements à risques. Mais, l'effet de la migration pourrait varier suivant le genre et la destination des migrations (Brockerhoff, 1998). Les migrants se déplaçant entre les aires urbaines et les migrantes en situation de mobilité entre les aires rurales seraient plus susceptibles que les non-migrant(e)s de s'engager dans des comportements à risques associés à l'infection par le VIH. Dans les aires rurales, les migrants urbains de retour seraient également plus susceptibles d'avoir des comportements à risques que les non-migrants. Cependant, cette analyse sur les migrations et comportements à risques accuse plusieurs limites : d'une part, elle n'identifie ni le rôle de la mobilité en 
tant que tel dans le mécanisme d'exposition aux risques ni le rôle des environnements et conditions sociales. D'autre part, elle n'opère pas de différenciation suivant les régions.

102 Ces éléments généraux permettent néanmoins d'esquisser des hypothèses sur la diffusion de l'épidémie à VIH au Kenya.

103 Les tendances étayent l'hypothèse selon laquelle le virus se serait diffusé des villes en direction des aires rurales de basse prévalence où les migrants masculins de retour auprès de leurs conjointes auraient été susceptibles d'avoir des relations sexuelles nonprotégées.

104 Cependant, si les comportements à risques ont été quantifiés, tel n'est pas le cas pour les risques de transmission et de diffusion du VIH en raison de l'absence de données de séroprévalence. Cette limite est susceptible de soulever un écueil pour l'interprétation, manifesté par la confusion entre comportements à risques et risque de transmission : le premier n'entraîne pas systématiquement le second mais les comportements à risques créent les conditions favorables à la transmission et à la diffusion du VIH.

Les migrations vers les aires urbaines se seraient traduites par une exposition différenciée aux risques suivant le genre. Les migrantes en milieu urbain, singulièrement celles qui sont originaires des autres aires urbaines, étaient moins susceptibles de s'engager dans des comportements à risques que les non-migrantes urbaines. Ceci remet en question l'argument selon lequel les femmes migrent vers les villes et ont recours au commerce sexuel comme stratégie de survie (Anarfi, 1993).

106 A contrario, les hommes qui se déplacent entre villes (qui représentent un quart des migrants masculins) étaient deux fois plus susceptibles de s'engager dans des comportements sexuels à risques comparés aux non-migrants. L'hypothèse a été posée que ces hommes sont essentiellement des cols blancs et des chauffeurs routiers avec des niveaux de revenus leur permettant de fréquenter des prostituées (Brockerhoff, 1998). Cette tendance aurait démultiplié les risques de transmission du VIH en milieu urbain (Brockerhoff, op. cit) en raison de la prédominance des migrations masculines vers les villes et du multi-partenariat sans recours au préservatif plus fréquent parmi les migrants $(29 \%)$ comparés aux non-migrants $(6 \%)$. Les comportements à risques selon les migrants entre villes seraient donc particulièrement marqués.

107 S'agissant des migrations urbaines-rurales, au regard des KDHS 1993 et 1998, 47 \% des migrantes de retour et $38 \%$ des hommes étaient susceptibles de s'engager dans des comportements sexuels à risque, soit des taux supérieurs aux non-migrants. Ces indicateurs accréditent encore l'idée selon laquelle les migrations circulaires ou les migrations urbaines-rurales ont favorisé la diffusion du VIH/sida en milieu rural. L'évolution de la prévalence au VIH en milieu rural a en effet connu un pic à la fin des années 1990 après avoir atteint son point d'acmé dans les villes. Cependant, les migrations circulaires vers les campagnes qui s'opèrent à une fréquence difficile à déterminer ne semblent pas avoir entrainé de façon homogène dans l'intérieur du pays une flambée de l'épidémie à VIH comparable à celle connue dans les villes ${ }^{4}$. Néanmoins, des populations de femmes enceintes dans quelques sites ruraux étudiés ont affiché des taux de prévalence équivalents à ceux observés dans des grandes villes, notamment à Chulaimbo - dans le district de Kisumu (37,3 \% en 1998) ou encore Usigu, localisé dans le district de Bondo dans la province de Nyanza (33 \% en 1999). Mais les différences de prévalence entre sites ruraux reflètent les disparités régionales constatées au Kenya: 
les centres de dépistages situés dans la Nyanza Province présentent des taux prévalence très élevés comparés à la moyenne nationale dans les localités rurales.

Au total, le rôle exact de la migration dans la diffusion et la distribution hétérogène du VIH/SIDA au Kenya est difficile à identifier. Néanmoins, il semble que les migrations aient véhiculé le virus des principales aires urbaines vers les aires rurales. En outre, certaines formes de mobilités liées au travail - notamment les déplacements des routiers - paraissent avoir clairement facilité la circulation du virus.

Cette difficulté à identifier le rôle de la migration sur la diffusion et la distribution hétérogène du VIH est liée à l'absence de donnée de séroprévalence ou d'incidence permettant d'apprécier avec précision l'état de la diffusion de l'épidémie dans la population générale en 1986 à l'échelle des villes, des localités rurales ou encore des districts. Selon une étude sur la distribution géographique de l'infection à VIH, la diffusion de l'épidémie tendrait à être uniforme et comprise entre 0 et $2 \%$. Nairobi se profile alors comme un des premiers foyers de développement de l'épidémie (Ouma, 1997) en raison de la présence du VIH dans des groupes noyaux tels que les prostituées. Mais il ne peut être exclu que le virus ait été présent dès le milieu des années 1980 dans la province du Nyanza, en raison de la proximité géographique de la région avec les premiers foyers de l'épidémie en Afrique Subsaharienne, localisés dans le district de Rakai en Ouganda.

La distribution de la prévalence du VIH dans les sites du réseau Sentinelle en 1990 et l'itinéraire supposé de la diffusion du VIH

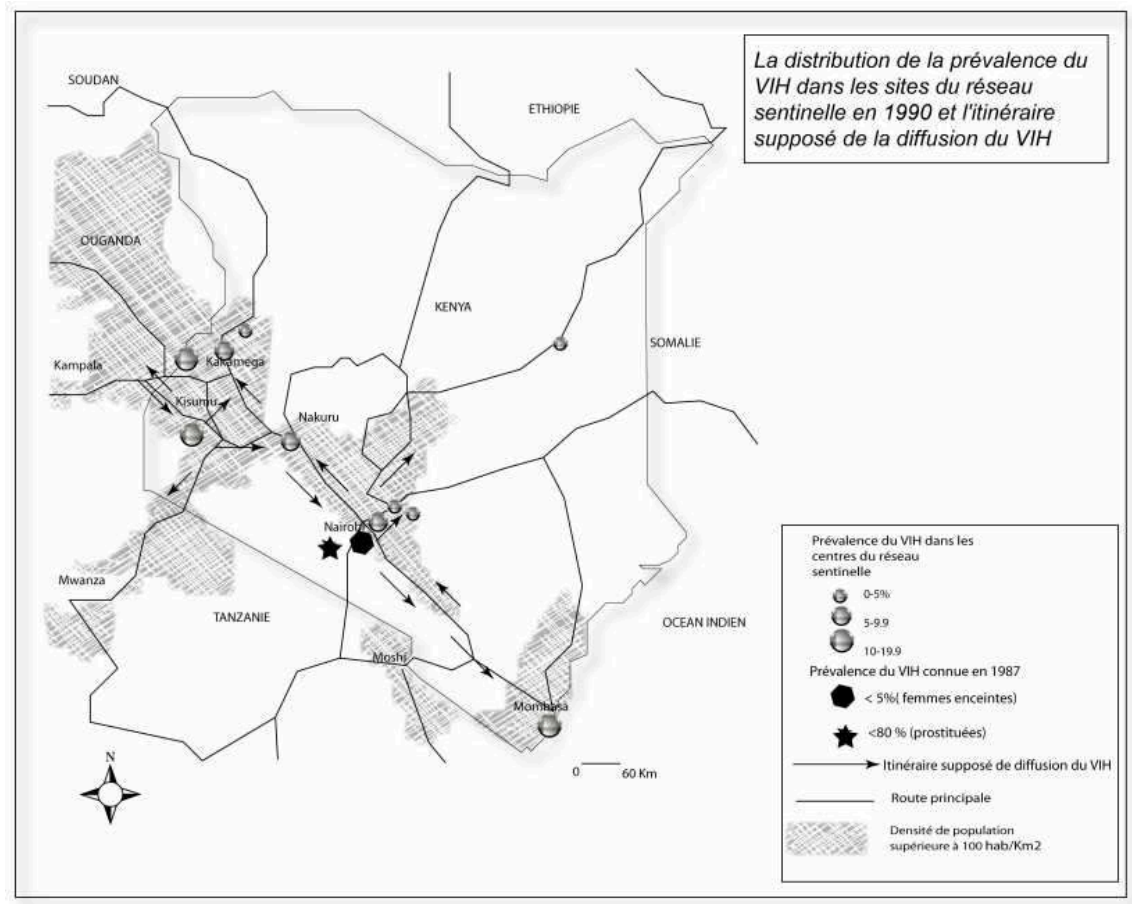

Cartographie: M. Miralles

110 En 1987, la prévalence au VIH dans une cohorte de femmes enceintes dans la capitale s'élève à 2,65 \%. Elle atteint $9 \%$ parmi les patients infectés par des MST et $80 \%$ chez les prostituées (ONUSIDA, 2003). La même année, les premiers cas de séropositivité sont 
également dépistés dans les districts de la province du Nyanza et à Mombasa. Dans la Central Province, les premiers cas sont détectés en 1988 puis en 1990 dans la Rift.

111 À partir des groupes noyaux - travailleuses du sexe, conducteurs -, l'épidémie se serait ensuite diffusée dans la population générale, des principaux pôles urbains du Kenya vers les villes secondaires et les campagnes par le truchement des mobilités géographiques.

In fine, au regard du paysage migratoire kényan et des différentes recherches sur l'épidémie à VIH au Kenya, il se pourrait que la migration n'ait pas contribué à la distribution hétérogène de l'épidémie à VIH entre la Nyanza Province et les autres régions du pays. Ces disparités semblent davantage liées à la prévalence de facteurs bio-médicaux - non-circoncision prédominante dans la Nyanza Province et Maladies sexuellement transmissibles.

113 En revanche, on peut émettre l'hypothèse que la faiblesse des migrations circulaires entre les régions semi-arides du Nord et les principales villes situées dans la partie médiane du pays ait freiné la diffusion du VIH dans les provinces nord; nord-est et nord-ouest du pays.

114 Le processus d'urbanisation alimenté par les migrations rurales urbaines s'est traduit par une croissance des densités humaines, et un bouleversement des environnements sociaux, économiques et sanitaires et économiques en milieu urbain. La conjonction de ces différents éléments a probablement contribué à la diffusion plus forte de l'épidémie dans les villes.

115 Les interrogations relatives à ces différents facteurs conjugués au rôle des déterminants socio-économiques dans l'exposition au VIH évoqué dans la deuxième partie de ce mémoire suggèrent que la distribution hétérogène de l'épidémie à VIH coïncide avec une pluralité d'environnements à risques, selon les milieux ruraux et urbains.

116 La conjonction d'analyses épidémiologiques ou relevant des sciences sociales nous a permis de cerner la pluralité de facteurs biologiques, comportementaux et sociaux susceptibles d'avoir favorisé la diffusion hétérogène de l'épidémie.

117 La distribution hétérogène de l'épidémie entre aires rurales, urbaines et à l'échelle infra-urbaine et l'éventail des facteurs en jeu dans les processus d'exposition au risque interroge quand à l'existence d'environnements favorisant la vulnérabilité a l'infection à VIH dans les bidonvilles de la capitale.

\section{La population des slums de Nairobi et la vulnérabilité à l'infection à VIH}

118 Les études récurrentes sur les liens entre pauvreté urbaine et exposition au VIH, urbanisation et forte prévalence invitent à une réflexion sur les composantes de l'environnement des slums susceptibles de favoriser la vulnérabilité à l'infection à VIHSIDA. L'intérêt de cette interrogation est conforté par les analyses contradictoires du KDHS 2003 et diverses enquêtes réalisées dans les slums de Nairobi : le premier a mis en évidence que les taux de prévalence les plus élevés pouvaient être observés au sein de populations urbaines aisées. Cependant, le taux de prévalence du VIH était estimé en 2005 a $15 \%$ (6-7\% a l'échelle nationale) dans le slum de Kibera tandis qu'une étude 
réalisée par l'African Population and Health Research Center indiquait qu'entre janvier 2003 et décembre 2005 la tuberculose et l'infection a VIH/SIDA étaient lies à l'origine de plus de $50 \%$ des décès au sein de la population âgée de 50 ans et plus, dans les slums de Korogocho et Viwandani.

Pour plusieurs auteurs, les slums se profilent, en effet, comme des révélateurs des crises urbaines qui se manifestent également sur le plan sanitaire. En effet, ces lieux cumulent la grande jeunesse de leur population, le chômage, la pauvreté, la précarité des conditions de logements, les sous-encadrements et sous-équipements, la violence, tous facteurs, qui conditionnent l'épidémiologie du sida (Amat-Roze, 2003). Quels sont les facteurs connus et conditions de vie favorisant la vulnérabilité à l'infection à VIH dans les slums de Nairobi?

\subsection{Les liens entre environnement et vulnérabilité à l'infection à VIH- SIDA}

L'effet des conditions de vie sur la vulnérabilité à l'infection à VIH ainsi que le rôle joue par les déterminants socioculturels et économiques sur l'exposition au risque a été suggéré par plusieurs études.

Leur enjeu a été de dévoiler les contextes dans lesquels se développent les comportements individuels, et notamment les conditions et contraintes au sein desquelles les décisions des personnes sont de fait prises dans la vie quotidienne, notamment en milieu urbain.

122 J. Benoist rappelait l'intérêt de cette problématique dans les années 1990 : « en observant que les modes d'organisation de la famille et les formes de vie amoureuse sont en pleine restructuration dans les milieux urbains africains, et on peut penser que certains changements comportementaux pourraient se faire assez rapidement pour contrer l'épidémie, si la situation économique des citadins africains n'était, par ailleurs, désastreuse un peu partout " (J. Benoist et A. Desclaux, 1996, p. 23).

Jean Benoist (Benoist, Desclaux, 1996) évoque l'intérêt de se concentrer sur les espaces sociaux (maisonnées, voisinage, lieu de travail.). Les recherches anthropologiques peuvent ainsi mettre en évidence le monde tel que le vivent les individus, les conditions concrètes de vie des gens de même que les systèmes de valeurs en référence auxquels les personnes agissent.

Cette préoccupation des recherches anthropologiques relative aux conditions de vie des individus est illustrée par des recherches réalisées dans des lieux spécifiques où se concentrent des populations dites vulnérables.

C. Campbell (Campbell, 1997) a ainsi montré, en Afrique du Sud, que les comportements sexuels des travailleurs migrants qui vivent très confinés sur les sites d'exploitation minière sont liés à leur identité sociale, marquée par leur condition de vie et de travail. Une étude menée (Vernazza-Licht et al., 2001) dans une plantation d'hévéas au Cameroun, présentée comme un véritable «village», fait apparaître la mobilité sexuelle comme un facteur aggravant le risque de contamination par le VIH. Ces quelques exemples tendent à prouver que l'implantation d'une industrie induirait un risque de changements sociaux, notamment la modification des normes matrimoniales et le recours à la prostitution. 
Une autre enquête, en République du Congo a mis en évidence des risques spécifiques de propagation de l'épidémie de VIH/SIDA selon une typologie d'environnements urbains et sociaux. (Enel, 2004).

Les données de cette enquête anthropologique qualitative menée dans cinq villes congolaises ont mis notamment en lumière que l'industrialisation autour du commerce du bois a aggravé la vulnérabilité des femmes car elle s'est développée sans prise de conscience des conséquences du fort pouvoir d'achat qu'elle entraîne : "Aucun gardefou, comme la promotion de l'épargne salariale ou de l'accès à la propriété ou à des terres cultivables, le regroupement familial, le développement des emplois féminins, n'a été dressé pour prévenir les comportements à risques ". L'absence de contrôle social due à la création ex nihilo d'agglomérations autour de sites forestiers a entraîné de nouveaux modèles de comportements, notamment la mobilité, la consommation abusive d'alcool, le multipartenariat sexuel et la prostitution. Une des conclusions de cette enquête est que l'environnement industriel de l'agglomération de Pokola suscite un risque accru de transmission du VIH, car les structures sociales induites par cette industrie favorisent une vulnérabilité par rapport au VIH.

À l'aune de ces différentes analyses, nous posons l'hypothèse que les slums de Nairobi se profilent comme des environnements dont les composantes et les conditions de vie favorisent la vulnérabilité ont l'infection à VIH.

\subsection{La population des slums, leur environnement de vie et la vulnérabilité a l'infection à VIH ? Éléments d'analyse et pistes de recherche}

Des discussions de groupe dirigées réalisées dans les bidonvilles de Kibera, Majengo, Kahawa North et Embakasi (Ezeh et al., 2003) mettent l'accent sur la misère et le désespoir qui caractérisent la vie des bidonvilles et sur le rôle essentiel de ces conditions de vies dans les comportements sexuels à risques. La position économique des femmes dans les bidonvilles serait de manière générale plus précaire que celle des hommes. Les frustrations et le chômage masculin entraîneraient une consommation d'alcool et de drogues (Mugisha et al, 2004) alors que la responsabilité de subvenir aux besoins de la famille et à l'alimentation des enfants incomberait aux femmes, dont l'entrée dans la vie adulte et le rôle seraient limités à celui de mère et d'épouse (Nairobi Urban Research Integration Project, 2004).

130 La large proportion de migrants masculins, célibataires ou séparés de leurs épouses qui y résident constituerait également une autre caractéristique importante des bidonvilles. La séparation des épouses aussi bien que l'offre de prostitution faciliteraient la fréquentation masculine des professionnelles du sexe.

131 Dans le même temps, la rapide augmentation des femmes migrantes dans les bidonvilles et la faiblesse des opportunités économiques pousseraient une proportion importante d'entre elles vers le commerce sexuel.

"For instance, if a woman stays alone, that is she is a single mother with children, and she wants to buy her children milk, the only solution would be to look for someone with money. She sells her body and gets the 20 shillings to buy milk. " (Kibera, femmes 13-17). (Zulu et al. 2003, p 171) 
Le commerce sexuel se présente parfois comme une alternative pour subvenir aux besoins alimentaires dans un contexte ou l'usage du préservatif - parfois trop onéreux, de 1 à $2 \mathrm{KSh}$, quand il n'est pas accessible gratuitement :

"Especially, in this village, there are very many women who stay on their own without their husbands". "This other one has a man and he is working and he has his wife. She will go to this one because of poverty... and you hear that she is married. Because you slept hungry and you have a husband who is not working, you will go this one."

Kahawa North, female 50+. (Zulu op.cit)

Leurs responsabilités de chefs de famille et le coût de la vie contraindraient les femmes à des comportements sexuels à risques. Des entretiens réalisés (Miralles, 2005) entre août 2004 et novembre 2005 accréditent cette assertion. Sur plus d'une trentaine de femmes interviewées dans le cadre d'entretiens semi-directifs sur leur relation avec leur région d'origine dans les quartiers de Gatwikira et Kianda à Kibera, 28 étaient séropositives et sous traitement ARV avec MSF Belgique. Vingt d'entre elles étaient originaires de la Nyanza Province et de la Western Province et étaient chefs de famille, suite au décès du partenaire ou à une séparation :

M. : «I've been living here for 4 years. It's changa' $a^{5}$ and men coming from my home area who come to have fun during the day... This is the way I live. »

L'exposition au risque VIH des habitants vivant dans les bidonvilles de Nairobi a été au cœur d'une analyse quantitative portant sur les comportements à risques à Nairobi à partir des questionnaires des KDHS 1993 et 1998 (APHRC, 2002). Cette étude met en relation les espaces des bidonvilles avec une forte prévalence des comportements à risques. Sur un échantillon de 413 femmes interrogées dans les bidonvilles, $75 \%$ déclaraient avoir grandi dans un village et avaient par la suite migré vers Nairobi. L'âge moyen de la première relation sexuelle dans cette population se situait à 15,6 ans contre 17,6 pour les femmes ne résidant pas dans les bidonvilles. $9 \%$ des résidentes des bidonvilles avaient eu des relations sexuelles multiples contre $4 \%$ pour les nonrésidentes. Parmi les femmes mariées, les habitantes du bidonville étaient plus susceptibles de pratiquer le multi-partenariat que les femmes ne résidant pas dans le bidonville.

Le nombre moyen de partenaires sexuels s'élevait à 1,23 pour les résidentes des bidonvilles et 1,06 pour les non-résidentes des bidonvilles. En outre, les femmes, chefs de famille, avaient plus de partenaires sexuels que celles qui ne l'étaient pas. La proportion de femmes qui avaient déjà utilisé des préservatifs était moins importante dans les bidonvilles que parmi les autres résidentes urbaines (10\% contre $27 \%$ ). Dans cette perspective, le KDHS 2003 indique que l'usage du préservatif est fortement associé à un niveau d'instruction élevé mais aussi que les femmes se voient plus fréquemment dénier son usage parmi les populations à très faible revenu et dans la classe d'âge 15-19 ans. Cette tendance coïncide avec une enquête de terrain réalisée en mars 2007 à Kibera: sur les 30 femmes interrogées, une majorité d'entre elles déclarait «ne pas pouvoir demander à leur conjoint » d'utiliser un préservatif même si elles le désiraient.

D'autres enquêtes (Voeten, 2000) portant sur la qualité de l'éducation à la santé étayent cette analyse, notamment une recherche réalisée dans 142 "structures de soins" implantées dans les slums de la capitale, en s'appuyant sur des entretiens avec 165 soignants et 441 patients souffrant de MST suivi par ces structures.

Parmi les différentes observations effectuées, il a été noté que la promotion du préservatif était insuffisante (dans $60 \%$ des cas). Les cliniques publiques étaient 
généralement les plus performantes tandis que les pharmacies et les cliniques de mission avaient les actions les plus insuffisantes dans ce domaine, en matière de promotion du préservatif.

Les connaissances et le recours aux préservatifs étaient encore moins plus faibles parmi les patients visités à leur domicile.

L'insuffisance de la promotion du préservatif a été mise en évidence par une autre enquête consacrée aux praticiens traditionnels.

ur les 16 entretiens réalisés dans 4 bidonvilles de Nairobi avec des soigneurs traditionnels, tous reconnaissaient que les MST étaient transmises sexuellement et identifiaient les principaux symptômes. Cependant, en matière de thérapies, Witchdoctors et les herbalistes dispensaient des traitements avec des herbes pour une moyenne de 7 jours, alors que les Spiritual Healers privilégiaient la prière. 13 Healers préconisaient l'abstinence sexuelle durant le traitement. Tous les soigneurs préconisaient de nouvelles visites tandis que 13 d'entre eux en cas de persistance des symptômes suggéraient de se rendre vers des structures de soins privées ou publiques.

Ces enquêtes sur l'utilisation du préservatif peuvent être mises en perspective avec l'hostilité manifestée par de nombreux leaders religieux et politiques.

En effet, au cours des séminaires organisés par MSF Belgique à Kibera à destination des responsables religieux, il est fréquent d'entendre ceux-ci assimiler l'usage du préservatif à un " pêché ».

143 En 1996, le leader de l'Église catholique au Kenya, le Cardinal Maurice Otunga conduisit des centaines de fidèles à Uhuru Park pour brûler des préservatifs : il ajouta que le sida pouvait être évité avec l'abstinence. Dans d'autres pays - en Ouganda, en Tanzanie -, des campagnes préconisaient la fidélité ainsi que l'utilisation du préservatif.et l'épidémie marqua un recul (Ogot, 2004).

$144 \mathrm{Au}$ total, les femmes et hommes qui ont recours au commerce sexuel, aux relations non-protégées, aux relations précoces, semblent proportionnellement plus représentés dans les bidonvilles de la capitale, peuplés majoritairement de migrant(e)s.

Les comportements à risques mais aussi certains facteurs favorisant la transmission non-usage du préservatif - pourraient procéder de situations de vulnérabilité associables au contexte (faiblesse des ressources économiques et inégalités de genre qui peuvent entraîner une plus grande dépendance économique des femmes) mais aussi de vulnérabilités inhérentes à certains sous-groupes de population -, jeunes, groupes singularisés par un faible niveau d'instruction.

146 Au-delà des difficultés d'accès aux ressources économiques et des bouleversements des modèles matrimoniaux suscités par les migrations, d'autres caractéristiques pourraient contribuer à faire des bidonvilles des environnements, favorisant la vulnérabilité à l'infection à VIH, notamment la faiblesse des encadrements sanitaires pour prévenir et traiter des MST qui favorisent la transmission du virus, l'insécurité et les violences récurrentes de milices dans certains slums, quand elles se traduisent par des violences sexuelles, les inégalités de genre, ainsi que les discours de certains leaders religieux et pratiques de soigneurs traditionnels bien que leur effet sur les comportements à risques soit, pour ces derniers, difficile à apprécier. 


\section{Conclusion} de populations.
Cette réflexion a tenté de mettre en lumière l'apport de l'analyse géographique à la compréhension de l'hétérogénéité spatiale et sociale de l'épidémie à VIH au Kenya.

La propagation du virus au sein de la population est le produit d'une conjonction d'éléments ayant au trait au comportement sexuel, à des facteurs biomédicaux qui accélèrent sa transmission ainsi qu'à des facteurs structurels ou conjoncturels. Les types de comportements et certains facteurs biomédicaux (la non-circoncision) peuvent être sous-tendus par l'éventail des contextes sociaux, économiques et culturels qui caractérisent la société kényane, suivant les villes, les campagnes, les régions, ou les quartiers d'une même ville. La vulnérabilité des populations est susceptible d'avoir été accentuée par plusieurs phénomènes telle que l'urbanisation explosive alimentée par les migrations rurales-urbaines, et dont les conséquences négatives ont été multiformes: encadrements sanitaires déficients; difficultés d'accès aux ressources économiques ; bouleversement des modèles matrimoniaux traditionnels.

Familière de quelques-uns de ces phénomènes, la géographie peut offrir des prismes de recherche complémentaires aux autres sciences, dans une approche de l'épidémie qui relève de l'écologie humaine : en effet, concepts et méthodes géographiques peuvent contribuer à localiser et identifier les combinaisons de facteurs de différentes natures et de circonstances susceptibles dans un lieu donne - slums, régions, ville avec une fonction de carrefour routier - d'avoir favorisé la diffusion hétérogène de l'infection a VIH ou dans une autre perspective d'accentuer la vulnérabilité a l'infection de groupes

L'analyse géographique peut aussi contribuer à dresser des typologies d'environnements à risques suivant la distribution géographique des facteurs mis en évidence par différentes recherches scientifiques.

Ainsi, on peut émettre l'hypothèse qu'il existe au Kenya une pluralité d'environnements favorisant la vulnérabilité à l'infection à VIH si l'on considère la multiplicité et la diversité des facteurs d'exposition et de transmission selon les lieux jalonnant les principaux axes routiers, les bidonvilles ou encore dans l'Ouest du pays. Cette hypothèse sur une pluralité d'environnements favorisant la vulnérabilité à l'infection à VIH et sa transmission pourrait être corroborée par un traitement statistique et cartographique des informations collectées dans les KDHS 1998 et 2003. Ces recensements démographiques et sanitaires contiennent en effet des informations sur la prévalence au VIH (KDHS 2003) ainsi que sur les facteurs d'exposition et de transmission associés au virus et les coordonnées géoréférencées des sites d'enquête en milieu rural et urbain, à différentes échelles. In fine, les typologies obtenues pourraient être représentées par des cartes et favoriser une localisation et une hiérarchisation des actions à entreprendre en matière de prévention et d'offres de soins.

Cette lecture géographique peut aussi favoriser une meilleure compréhension des facteurs contextuels généraux. Leur prise en compte est une voie indispensable pour sortir d'une épidémie qui provoque une aggravation de la pauvreté, la rupture des services sociaux et la détérioration de la matrice de renouvellement de la société.

Cette ouverture sur le rôle des facteurs contextuels a entraîné en 1995 la dissolution du Programme global du sida de l'OMS et la création d'ONUSIDA, un programme conjoint 
des Nations Unies qui associe désormais neuf organisations internationales dans la mise en œuvre d'une approche multisectorielle de l'épidémie du sida.

\section{BIBLIOGRAPHIE}

African Population and Health Research Center (APHRC). (2002). Population and health dynamics in Nairobi's informal settlements. Nairobi : APHRC (185 p.).

Anarfi, J. (1993). « Sexuality, Migration and AIDS in Ghana: a socio-behavioral study. » Health Transition Review 3: 45-67.

Anderson R.M., May R.M., Boily M.C., Garnett G.P., Rowley J.T. (1991). « The spread of HIV-1 in Africa: sexual contact patterns and the predicted demographic impact of AIDS. » Nature 352 : 581-9.

Amat-Roze, J.M. (1989). « HIV-infection and AIDS in sub-Saharan Africa: factors of regionalization. » Cahiers d'Outre-mer 42: 333-55.

Amat-Roze J.M. (1993). « Geographical inequalities of HIV-infection and AIDS in sub-Saharan Africa. » Social Science and Medicine 36 : 1247-56.

Amat-Roze, J.M. (2003). « L'infection à VIH/Sida en Afrique subsaharienne, propos géographiques. » Hérodote 4/111 : 117-155.

Bailey, R.C., Moses, S., Parker, C., et al. (2007). « Male circumcision for HIV prevention in young men in Kisumu, Kenya : a randomised controlled trial. » The Lancet 369 : 643-56.

Benoist, J., Desclaux, A. (eds) (1996). Anthropologie du sida. Bilan et perspectives. Paris : Karthala (381 p.).

Brockerhoff, FM., Biddelcom, A. (1998). « Migration, Sexual Behaviour and HIV Diffusion in Kenya. » International Migration Review 31 : 833-56.

Brown, R.C., Brown, J.B., Ayowa, O. (1993). « The use and physical effects of intravaginal substances in Zairean women. » Sexually transmitted Diseases, 20 : 96-9.

Buve, A., Carael, M., Hayes R.J., et al. (2001). « Multicentre Study on Factors Determining Differences in Rate of Spread of HIV in Sub-Saharan, Africa: Methods and Prevalence. » AIDS 15 : S5-S14.

Campbell, C. (1997). « Migrancy, masculine identities and Aids: the psychosocial context of HIV transmission on the South African gold mines. » Social Sciences and Medicine 4 : 273-81.

Decosas, J. (1995). Migration and AIDS, The Lancet 346 : 826-8.

Desgrées du Loû A., Ferry, B. (eds.) (2006). Sexualité et procréation confrontées au Sida dans les pays du sud. Paris : les collections du CEPED.

Eliot, E. (2000). Éléments et propositions pour une géographie du VIH/SIDA en Inde. Espace, Populations, Sociétés $2: 179-94$. 
Enel, C., Querre, M. (2006). « La vulnérabilité des femmes face au VIH en République du Congo : les composantes des environnements à risques. » In : A. Desgrées du Loû, B. Ferry (eds). Sexualité et procréation confrontées au Sida dans les pays du Sud : 59-78. Paris : CEPED.

Farmer, P. (1996). Sida en Haïti. La victime accusée. Paris : Karthala (409p.).

Gould P. (1993). The Slow Plague: a geography of the Aids epidemic. Cambridge: Blackwell Press.

Grosskurt, H., Mosha, F., Todd, J. (1995). « Impact of improved treatment of sexually transmitted diseases on HIV infection in rural Tanzania: randomised controlled trial. » The Lancet 346 : 530-6.

Hunter, D.J., Maggwa, B.N., Mati, J.K., Tukei, P.M., Mbugua, S. (1994). « Sexual behaviour, sexually transmitted diseases, male circumcision and risk of HIV infection among women in Nairobi, Kenya. » AIDS 8 : S93-S99.

Hargreaves, R., Morison, L., Chege J., et al. (2002). « Socioeconomic and risk of HIV infection in an urban population in Kenya. » Tropical medicine and international health 9: 793-802.

Jackson, D., Rakwar, J., Bwayo, J., et al. (1994). « HIV/STD Incidence among Truck Drivers in Mombasa, Kenya: Role of Circumcision Status. » Tenth International Conference on AIDS, Yokohama, Japan Abstract 409C, 8 : 7-12.

Jackson, D., Ngugi, N., Plummer, F.A., et al. (1999). « Stable Antenatal HIV-1 Seroprevalence with High Population Mobility and Marked Seroprevalence Variation among Sentinel Sites. » AIDS 5 : S583-S589.

Lalou, R., Enel, C., Querre M., Rogier, C. (2006). « Les épidémies du VIH/SIDA en République du Congo dans un contexte d'après-guerre : disparités géographiques et pluralité des environnements à risque. » In : A. Desgrées du Loû, B. Ferry (eds). Sexualité et procréation confrontées au Sida dans les pays du Sud : 59-78. Paris : CEPED.

Lydié, N., Robinson, N.J. (1998). « Migration and HIV-AIDS in West and Central Africa. » International Migration ; 4 : 469-511.

Lydié, N. (2001). « L'épidémie du VIH/SIDA en Afrique: état des lieux. » La Documentation FrançaiseAfrique contemporaine $3: 73-87$.

Miralles, M. (2005). « Migrations et sida au Kenya : état des connaissances et perspectives de recherches. » Annuaire de l'Afrique Orientale 2005 : 299-346. Nairobi : IFRA; Paris: L'Harmattan.

Morris, C. (2004). Hot Spot Mapping of the Mombasa-Kampala Highway, University of Manitoba, document PowerPoint.

Morris, M., Kretzchmar, M. (1997). Concurrent partnerships and the spread of HIV, AIDS 11 : S641S648.

McCutchan, F.E. (2000). « Understanding the genetic diversity of HIV-1. » AIDS 3 : S31-S44.

MSF Belgium (2006). Kenya annual report, working paper.

OGOT B (2004). « Politics and the Aids Epidemic in Kenya 1983-2003. » Anyange Press LTD, Kisumu : 1-127.

Ouma, V. (1997). « A spatio-temporal analysis of HIV/AIDS diffusion in Kenya 1986-1993. » Urbanrural studies:116-40.

Prothero, R.M. (1994). « Forced movements of population and health hazards in tropical Africa. » International Journal of Epidemiology 6 : 259-67.

Prothero, R.M. (1996) « Migration and Aids in West Africa. » Geography 81: 353-74. 
Rémy, G. (1993). « L'espace épidémiologique de l'infection par le virus de l'immunodéficience humaine VIH2 en Afrique Subsaharienne. » Médecine Tropicale ; 4 : 511-6.

Rémy, G. (2002). Mobilité des personnes et diffusion du sida en Afrique de l'Ouest. L'espace géographique $3: 253-63$.

Republic of Kenya, Kenyan Demographic and Health Survey. Nairobi: Central Bureau of Statistics ; $1993 ; 1998 ; 2003$.

Rhein, C. (2003). L'écologie humaine-discipline chimère. Sociétés contemporaines 49-50 : 167-90.

Ruffié, J., Sournia, J.-C. (1999). Les épidémies dans l'histoire de l'homme. Paris : Flammarion (302 p.).

Tyndall, M., Kidula, W., Sande, J., et al. (1998). «Vulnerability to HIV among Disadvantaged

Women in Kenya: Implications for Interventions. » $12^{\text {th }}$ World AIDS Conference. 7/3, Abstract 23449.

UNAIDS. Kenya Epidemiological Fact sheets, 2003, 2005, 2006.

US Bureau of the Census. (2003). HIV/AIDS in Africa. Health Studies Branch, International programs center, Population division, Research Note Number 20.

Vernazza-Licht, N., Ageba, S.C., Bley, D., Pagezy, H. (2001). « Sida et gestion de la santé des travailleurs dans une plantation au Sud-Cameroun. » Le journal du sida et de la démocratie sanitaire $138: 29-34$

Voeten H. (2000). «Effectiveness of STD case management in Nairobi, Kenya : design. » Proceedings of the XIII International Conference, Durban, South Africa, July 9-14 : 69-74.

Williams, B., Gouws, E., Lurie, M., Crush, J. (2002). « Spaces of vulnerability : Migration and HIV/ AIDS in South Africa. » Migration Policy; 24 (42 p).

Weiss, H.A., Quigley, M., Hayes, R.J. (2000). « Male circumcision and risk of HIV infection in subSaharan Africa : a systematic review and meta-analysis. » AIDS 14 : S2361-70.

Wang C.C., Kreiss J.K., Reilly M. (1999). « Risk of HIV infection in oral contraceptive pill users: a meta-analysis. » Journal of Acquired Immune Deficiency Syndromes 21: 51-8.

Zulu, E., Dodoo, F., Ezeh, A. (2003). « Urbanization, poverty, and sex: Roots of risky sexual behaviours in slum settlements in Nairobi. » APHRC Research Report. 167-73.

\section{NOTES}

1. La prévalence peut être définie comme la proportion d'individus infectés dans une population donnée à un moment T. L'incidence est le nombre de nouveaux cas d'une pathologie observés pendant une période et pour une population déterminées.

2. On ne peut affirmer que les données de séroprévalence de l'ONUSIDA censées représenter l'évolution du SIDA au sein de la population rurale reflètent la physionomie de l'épidémie en milieu rural dans la mesure où l'ONUSIDA estime la prévalence de la population vivant dans cet environnement à partir de sites localisés dans des villes petites et moyennes (Thika, Mbale...). Autrement dit, il est possible que les populations dépistées vivent dans ces unités urbaines et non dans des villages ruraux. Le critère de distinction géographique de l'ONUSIDA distinguant les " grandes aires urbaines » telles que Nairobi et Kisumu et les sites localisés "hors des grandes aires urbaines" ne semble donc pas totalement opératoire pour donner une appréciation juste de l'épidémie en milieu rural et urbain. 
3. Dans le groupe des facteurs biologiques, nous intégrons par exemple le type de virus. La non-circoncision pourrait être considérée comme un facteur relevant des catégories biologiques et écologiques dans la mesure où ce déterminant qui favorise la transmission biologique du virus est aussi lié à l'environnement socio-culturel de l'individu. Les MST pourraient aussi être considérées comme un facteur avec une dimension écologique dans la mesure où leur diffusion est facilitée par l'absence de dispositifs de prévention dans l'environnement social de l'individu.

4. Il est difficile de présenter une évolution des données de séroprévalence en milieu rural, dans la mesure où les données lacunaires grèvent les possibilités d'appréciation.

5. Le chang'aa est un alcool originaire de la Nyanza Province frequemment distillé dans les slums.

INDEX

Index géographique : Kenya

\section{AUTEUR}

MATTHIEU MIRALLES

Doctorant en géographie à l'Université Bordeaux 3. 